\title{
Is individual cognitive behavioral therapy as effective as antidepressants in patients with major depressive disorder?
}

\author{
2021
}

Dams, Travis J. MD; Dhesi, Tajinder S. MD

\begin{abstract}
Evidence-based Answer: Individual cognitive behavioral therapy is as effective as antidepressant medication in the treatment of major depressive disorder (SOR: A, consistent findings from two randomized controlled trials).
\end{abstract}

A 2017 randomized controlled trial $(\mathrm{N}=344)$ evaluated the effectiveness of cognitive behavioral therapy (CBT) versus pharmacotherapy in the treatment of major depressive disorder (MDD). ${ }^{1}$ Patients (mean age 40 years old) who met the Diagnostic and Statistical Manual of Mental Disorders, Fourth Edition, Text Revision (DSM-IV-TR) criteria for MDD without psychotic features and received a score of 18 or greater at screening and 15 or greater at baseline on the 17-item Hamilton Depression Rating Scale (HAM-D) were included. Scores of seven or less indicate normal range or remission, and scores of 20 or greater indicate depression of moderate severity. Patients were randomized to receive 12 weeks of either CBT $(n=115)$, duloxetine $(n=115)$, or escitalopram $(n=114)$. The CBT group received individual twice weekly sessions for the first four weeks, followed by weekly sessions for the subsequent eight weeks. The pharmacotherapy groups received 12 weeks of either escitalopram 10 to $20 \mathrm{mg}$ daily or duloxetine 30 to $60 \mathrm{mg}$ daily. Of the 344 patients who were initially randomized into each group, 28 did not return for a postrandomization assessment and 234 patients met all the criteria in the per-protocol completer sample. A nonresponse to treatment was defined as a reduction of $30 \%$ or less from baseline

This is the author's manuscript of the work published in final edited form as:

Dams, T. J., \& Dhesi, T. S. (2021). Is individual cognitive behavioral therapy as effective as antidepressants in patients with major depressive disorder? Evidence-Based Practice, 24(5), 21-22. https://doi.org/10.1097/EBP.0000000000001075 
in HAM-D scores, a partial response was between a 30\% to $49 \%$ reduction, a response without remission was a $50 \%$ or greater reduction but still an overall score of seven or greater, and remission was defined as a score lower than seven. There was no significant difference in mean HAM-D reduction scores between the CBT, escitalopram, and duloxetine groups $(-9.8,-11.1$, and $-10.7 ; P=.42)$. Additionally, remission rates did not significantly differ between the CBT, escitalopram, and duloxetine $(44 \%, 44 \%$, and $52 \% ; P=.51)$. There was no significant difference between the three groups for the remaining categorical outcomes. Limitations included lower doses of duloxetine than what is commonly available, patients not blinded to their treatment assignment, a short duration of treatment monitoring, and use of individual $\mathrm{CBT}$ instead of group $\mathrm{CBT}$, which may be more available in certain locations.

A 2005 multicenter randomized, placebo-controlled trial $(\mathrm{N}=240)$ compared the efficacy of antidepressant medication with cognitive therapy in patients with moderate-to-severe depression. ${ }^{2}$ Patients were adults (mean age 40 years old) with moderate-to-severe depressive disorder. Diagnosis was based on the DSM-IV criteria with scores of 17 or higher on the HAM-D scale at a screen and baseline visit separated by at least seven weeks. Participants were excluded if they presented with other psychiatric conditions such as substance abuse deemed to require treatment, bipolar disorder, and other Axis I or II conditions that were deemed more pressing for treatment or whose course would be hindered by the treatment in the study. Patients in the pharmacotherapy group $(n=120)$ were prescribed paroxetine 10 to $20 \mathrm{mg}$ daily which was titrated up or down ( $\mathrm{min} 10 \mathrm{mg}$, max $50 \mathrm{mg}$ ) over the course of eight weeks until a significant reduction of symptoms was seen. Individual cognitive therapy $(n=60)$ sessions were held twice weekly for the first four weeks, once or twice weekly for the middle eight weeks, and once weekly for the last four weeks. The placebo group $(n=60)$ received placebo pills on the same schedule as the medication group but were offered medication at the eight-week mark. The primary outcome was a response, described as a modified 17-item Hamilton Depression Rating Scale (HDRS) score of 12 or less at 16 weeks. Scores of seven or less are considered within normal range or 
clinical remission, while a score of 20 or greater indicates moderate severity. Baseline HDRS score mean was 23 and did not significantly differ between the treatment groups. There was no significant difference between response rates for the cognitive therapy group and the pharmacotherapy group ( $58 \%$ vs $58 \%, P=.92$ ). The medication group and the cognitive behavioral group both demonstrated significant increased response rates compared to placebo. There were no harms identified during the study. 


\section{References}

1. Dunlop B, Kelley M, Aponte-Rivera V, et al. Effects of patient preferences on outcomes in the predictors of remission in depression to individual and combined treatments (PReDICT) study. Am J Psychiatry. 2017; 174(6):546-556. [STEP 2]

2. DeRubeis RJ, Hollon SD, Amsterdam JD, et al. Cognitive therapy vs medications in the treatment if moderate to severe depression. Arch Gen Psychiatry. 2005; 62:409-416. [STEP 2] 